\title{
Liquid-Gas Coexistence and Critical Behavior in Boxed Pseudo-Fermi Matter
}

\author{
Jan Tõke, Jun Lu, and W.Udo Schröder \\ Department of Chemistry, University of Rochester, Rochester, New York 14627
}

(Dated: November 25, 2018)

\begin{abstract}
A schematic model is presented that allows one to study the behavior of interacting pseudoFermi matter, locked in a thermostatic box. As a function of the box volume and temperature, the matter is seen to show all of the familiar characteristics of a Van der Waals gas, which include the coexistence of two phases under certain circumstances and the presence of a critical point.
\end{abstract}

PACS numbers: 21.65+f, 21.60.Ev, 25.70.Pq 


\section{INTRODUCTION}

The possibility that a liquid-gas phase transition in finite nuclei may manifest itself via copious production of intermediate-mass fragments (IMF) in energetic heavy-ion reactions has driven both, theoretical and experimental studies of nuclear multifragmentation over more than a decade. A prominent role in theoretical considerations is played by the concept of a freezeout configuration. [1, 2] This concept implies the existence of a definite volume, within which the system reaches a state close to thermal equilibrium. While the existence of an effective freezout volume may be debatable, the concept itself is useful for understanding the possible behavior of nuclear matter under various conditions. With such a didactic strategy in mind, and as an extension of earlier studies modelling the behavior of finite Fermi systems, [3, 4, 5] the present study considers pseudo-Fermi matter confined to a box of definite volume. It evaluates the isothermal behavior of such matter and its dependence on box volume and temperature. The utmost simplicity of the formalism allows one to gain insight into physical phenomena that may be obscured in more rigorous approaches.

\section{THEORETICAL FORMALISM}

The present study considers a scenario of nuclear pseudo-Fermi matter of mass number $A$, locked in a spherical box of a volume $V$ and kept at constant temperature $T$. In the proposed formalism, thermostatic properties of the matter are modelled by two equations, the isochoric caloric equation of state, and the zero-temperature equation of state. The equilibrium state of the system is then found as a function of $V$ and $T$, based on the requirement that the free energy of the system be minimal.

First, we consider homogeneous systems with constant density throughout their volume, i.e., systems in states where only one phase is present. The isochoric caloric equation of state for such a system is taken in a simple form adequate for low-temperature Fermi gases

$$
E_{\text {therm }}^{*}=a T^{2}
$$


where $E_{\text {therm }}^{*}$ is the thermal energy and $a$ is the level density parameter. The latter parameter is assumed to depend on matter density as

$$
a=a_{o}\left(\frac{\rho}{\rho_{o}}\right)^{-\frac{2}{3}}
$$

in accordance with the low-temperature Fermi gas model.

It is worthwhile keeping in mind, that for Fermi gases, Eq. 11 is a good approximation only for temperatures that are small compared to the Fermi energy, i.e., for $T<<E_{F}$. Notably, for diluted or very hot Fermi systems, with the matter density approaching zero, $\rho->0$, or for high temperatures, $T->\infty$, the caloric equation of state approaches asymptotically that of a classical gas:

$$
E_{\text {therm }}^{*}=\frac{3}{2} A T
$$

For the sake of simplicity and without loss of generality, the present study uses Eq. 1 over the full range of matter densities and temperatures considered. The term "pseudoFermi" matter is used to distinguish the matter considered here from true Fermi matter.

The second defining equation, the zero-temperature equation of state, expresses the compressional (potential) energy of the system as a function of the system volume or as a function of matter density. The present study adopts the harmonic approximation in the form used in the Expanding Emitting Source Model (EESM) [6], whereby the inmedium nucleonic (potential) energy changes quadratically with the relative deviation of the actual matter density from the ground-state density, by an amount

$$
\epsilon_{\text {compr }}=-\epsilon_{B}\left(1-\frac{\rho}{\rho_{o}}\right)^{2}
$$

In Eq. 廿, $\epsilon_{\text {compr }}$ and $\epsilon_{B}$ are compressional and ground-state binding energies per nucleon, respectively, and $\rho$ and $\rho_{o}$ are the actual and the ground-state matter densities, respectively. Equation 4 implies an effective ground-state incompressibility constant of $K_{o}=-18 \epsilon_{B}$. Assuming $\epsilon_{B}=-8 \mathrm{MeV}$, the effective incompressibility, including the effects of surface tension, equals $K_{o}=144 \mathrm{MeV}$. Note, that within the harmonic approximation, for infinite nuclear matter characterized by $\epsilon_{B} \approx-16 \mathrm{MeV}$, the incompressibility constant is $K_{o} \approx 288 \mathrm{MeV}$. The latter value places the present harmonic approximation 
"neutrally" between the currently considered limits of "soft" and "hard" equations of state for nuclear matter.

While the two defining equations 1 and 1 may be considered rather crude approximations, they do contain the essential physics responsible for first-order phase transitions and critical phenomena. Given these two equation, one can write expressions for all thermodynamic quantities characterizing the system, including the Helmholtz free energy $F$. The state of the system can then be found by minimizing the free energy, for any box volume $V$ and temperature $T$.

Based on Eq. 1, one can write for the entropy $S$ of a homogeneous system

$$
S=\int_{0}^{E *} \frac{1}{T} d \epsilon=2 \sqrt{a E^{*}}
$$

The free energy, $F$, for a homogeneous, single-phase system is given by

$$
F=E_{\text {total }}^{*}-S T=E_{\text {compr }}^{*}+E_{\text {therm }}^{*}-2 a T^{2}=E_{\text {compr }}^{*}-a T^{2} .
$$

In Eq. 6, for sake of simplicity, the free energy is given relative to a constant ground state energy of the system. Further, using Eqs. 2 and \&, Eq. 6 can be rewritten in a form revealing explicitly the important dependence on the matter density $\rho$, i.e.,

$$
F=E_{B}\left(1-\frac{\rho}{\rho_{o}}\right)^{2}-a_{o}\left(\frac{\rho}{\rho_{o}}\right)^{-\frac{2}{3}} T^{2}
$$

Eq. 7 allows one to write equations for the system pressure $p$ and the chemical potential $\mu$ as functions of volume (matter density) and temperature.

The pressure $p$ can be expressed generally as the negative partial derivative of the free energy $F$ with respect to volume $V$, at fixed number of nucleons $A$ and fixed temperature $T$, i.e.,

$$
p=-\left(\frac{\partial F}{\partial V}\right)_{A, T}=\frac{1}{A} \rho^{2}\left(\frac{\partial F}{\partial \rho}\right)_{A, T}
$$

Thus, for the case of pseudo-Fermi matter considered here, one obtains based on Eqs. 7 and 8

$$
p=2 \epsilon_{B} \rho_{o}\left(1-\frac{\rho}{\rho_{o}}\right)\left(\frac{\rho}{\rho_{o}}\right)^{2}+\frac{2}{3} \alpha_{o} \rho_{o}\left(\frac{\rho}{\rho_{o}}\right)^{\frac{1}{3}} T^{2},
$$

where $\epsilon_{B}$ and $\alpha_{o}$ are the binding energy per nucleon and the level-density parameter per nucleon, respectively. 
The chemical potential $\mu$ can be expressed generally as a partial derivative of free energy $F$ with respect to the number of nucleons $A$, taken at fixed volume $V$ and fixed temperature $T$, i.e.,

$$
\mu=\left(\frac{\partial F}{\partial A}\right)_{V, T} .
$$

Using Eqs. 7 and 10, and noting further that $\rho=A / V, E_{B}=A \epsilon_{B}$ and $a_{o}=A \alpha_{o}$, one obtains for the chemical potential

$$
\mu=\epsilon_{B}\left[1-4 \frac{\rho}{\rho_{o}}+3\left(\frac{\rho}{\rho_{o}}\right)^{2}\right]-\frac{1}{3} \alpha_{o}\left(\frac{\rho}{\rho_{o}}\right)^{-\frac{2}{3}} T^{2} .
$$

It is worth noting in Eq. 11] that, as a result of the requirement for $V$ to be constant, the magnitude of the chemical potential differs significantly from the value of the free energy per nucleon. For example, the contribution of thermal excitation to the chemical potential is only one-third of what constitutes the thermal part of the free energy per nucleon.

\section{LIQUID-GAS COEXISTENCE}

When confined to a thermostatic box, matter will eventually fill all of the available volume $V$ so as to minimize the free energy of the system. For non-interacting matter, the minimum free energy always corresponds to a uniform density distribution. This is generally not true for an interacting system. In particular, for interacting pseudo-Fermi matter at the minimum free energy, low-density and high-density phases coexist.

For a two-phase system of $A$ nucleons confined to volume $V$ at temperature $T$, the free energy can be expressed as a function of two variables, e.g., in terms of the volume of the gaseous phase, $V_{\text {gas }}$, and the number of nucleons contained in this phase, $A_{\text {gas }}$.

$$
F=F_{\text {gas }}+F_{\text {liquid }},
$$

Inserting for $F_{\text {gas }}$ and $F_{\text {liquid }}$ the expressions given by Eq. 7 for the corresponding numbers of nucleons $A_{\text {gas }}$ and $A_{\text {liquid }}$, respectively, one obtains

$$
F_{\text {gas }}=A_{\text {gas }} \epsilon_{B}\left(1-\frac{A_{\text {gas }}}{V_{\text {gas }} \rho_{o}}\right)^{2}-A_{\text {gas }} \alpha_{o}\left(\frac{A_{\text {gas }}}{V_{\text {gas }} \rho_{o}}\right)^{-\frac{2}{3}} T^{2}
$$




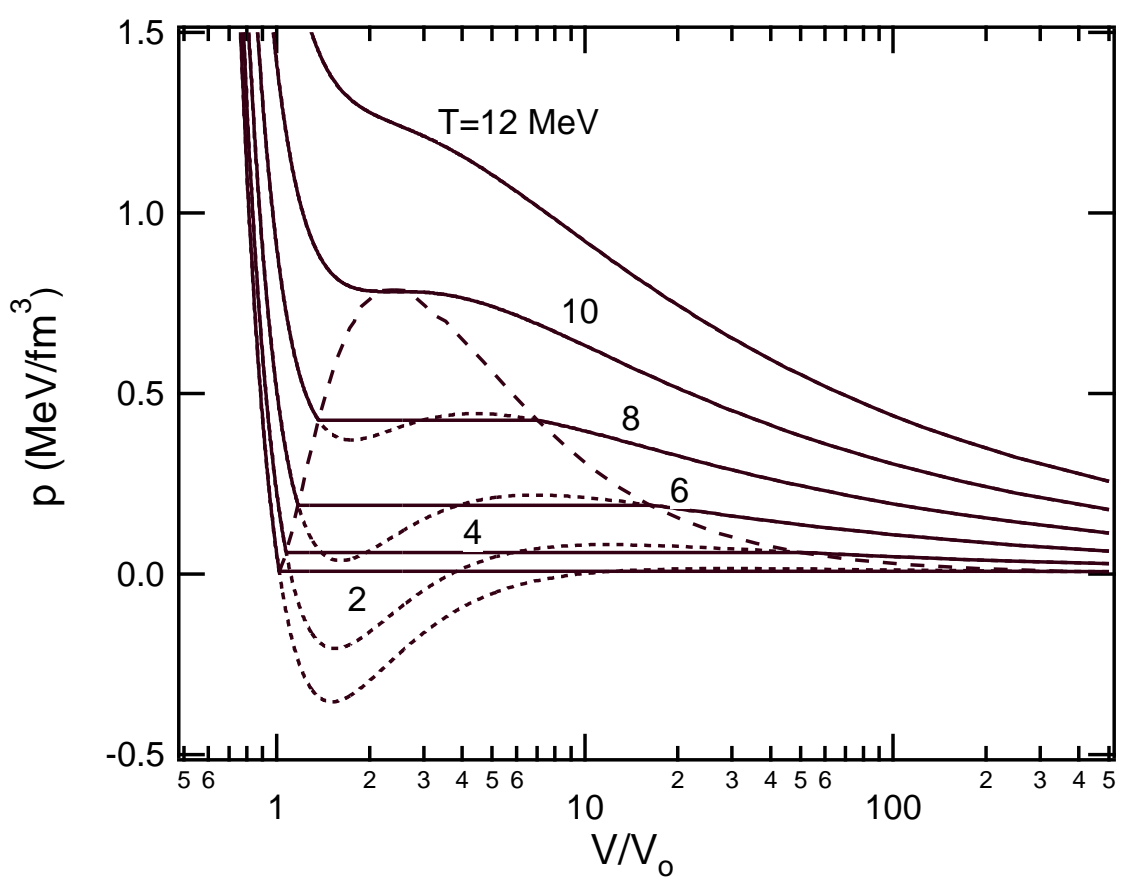

FIG. 1: Isotherms calculated for an ${ }^{197} \mathrm{Au}$-like system. Dotted lines illustrate isotherms for hypothetical single-phase matter, while the dashed line visualizes the boundary of the liquidgas coexistence domain.

and

$$
F_{\text {liquid }}=\left(A-A_{\text {gas }}\right) \epsilon_{B}\left[1-\frac{A-A_{\text {gas }}}{\left(V-V_{\text {gas }}\right) \rho_{o}}\right]^{2}-\left(A-A_{\text {gas }}\right) \alpha_{o}\left[\frac{A-A_{\text {gas }}}{\left(V-V_{\text {gas }}\right) \rho_{o}}\right]^{-\frac{2}{3}} T^{2}
$$

The condition for the minimum free energy can be expressed in form of two equations, reflecting requirements of dynamical and chemical equilibrium, respectively

$$
\left(\frac{\partial F}{\partial V_{\text {gas }}}\right)_{T, A_{\text {gas }}}=\left(\frac{\partial F_{\text {gas }}}{\partial V_{\text {gas }}}\right)_{T, A_{\text {gas }}}-\left(\frac{\partial F_{\text {liquid }}}{\partial V_{\text {liquid }}}\right)_{T, A_{\text {liquid }}}=p_{\text {gas }}-p_{\text {liquid }}=0
$$

and

$$
\left(\frac{\partial F}{\partial A_{\text {gas }}}\right)_{T, V_{\text {gas }}}=\left(\frac{\partial F_{\text {gas }}}{\partial A_{\text {gas }}}\right)_{T, V_{\text {gas }}}-\left(\frac{\partial F_{\text {liquid }}}{\partial A_{\text {liquid }}}\right)_{T, V_{\text {liquid }}}=\mu_{\text {gas }}-\mu_{\text {liquid }}=0 .
$$

Results of a numerical minimization of the free energy of a two-phase system are shown in Figs. 11- Fig. 1 presents system isotherms, as predicted by the present formalism, in a representation of system pressure $p$ versus system volume $V$. Note that Eqs. 13 and 14 represent single-phase states as special cases. A pure liquid/gas state is 


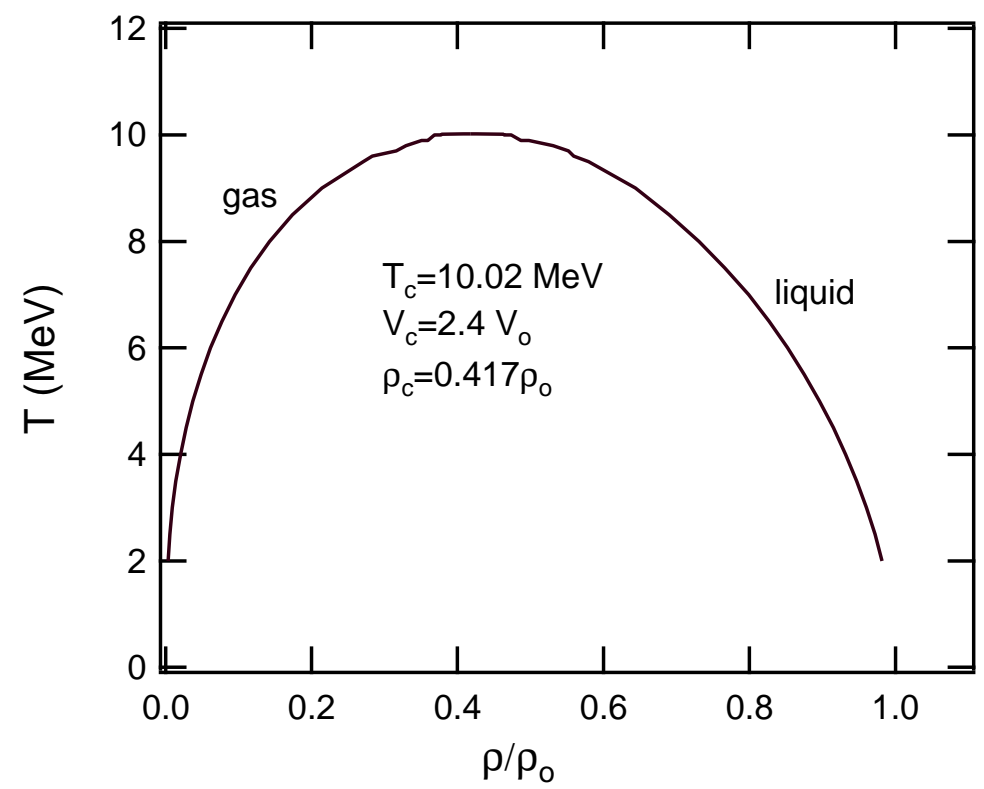

FIG. 2: Liquid-gas coexistence line in the temperature versus matter density representation, as predicted by the present formalism.

thus among the possible outcomes of the calculations with $A_{\text {gas } / \text { liquid }}=0$. As seen in Fig. 1, the isotherms feature segments representing both, pure liquid and pure gas and, notably, the liquid-gas coexistence "plateaus". The presence of coexistence plateaus does not come as a surprise as the harmonic interaction term of Eq. Thas the salient characteristics of a Van der Waals interaction.

It is worth noting that the coexistence plateaus in this calculation result naturally from the actual minimization of the free energy and are not obtained via the wellknown phenomenological Maxwell construct. Isotherms for hypothetical single-phase states are shown as dotted lines. These latter isotherms feature domains of spinodal instability characterized by negative compressibility. The dashed line in Fig. 11 illustrates the boundary of the liquid-gas coexistence domain. The "summit" point of this curve represents the critical point for the system and corresponds to a critical temperature of $T_{c} \approx 10.0 \mathrm{MeV}$. At temperatures higher than $T_{c}$, the system can reside only in a single-phase, vapor state.

A different representation of the liquid-gas coexistence line is illustrated in Fig. 2. In 


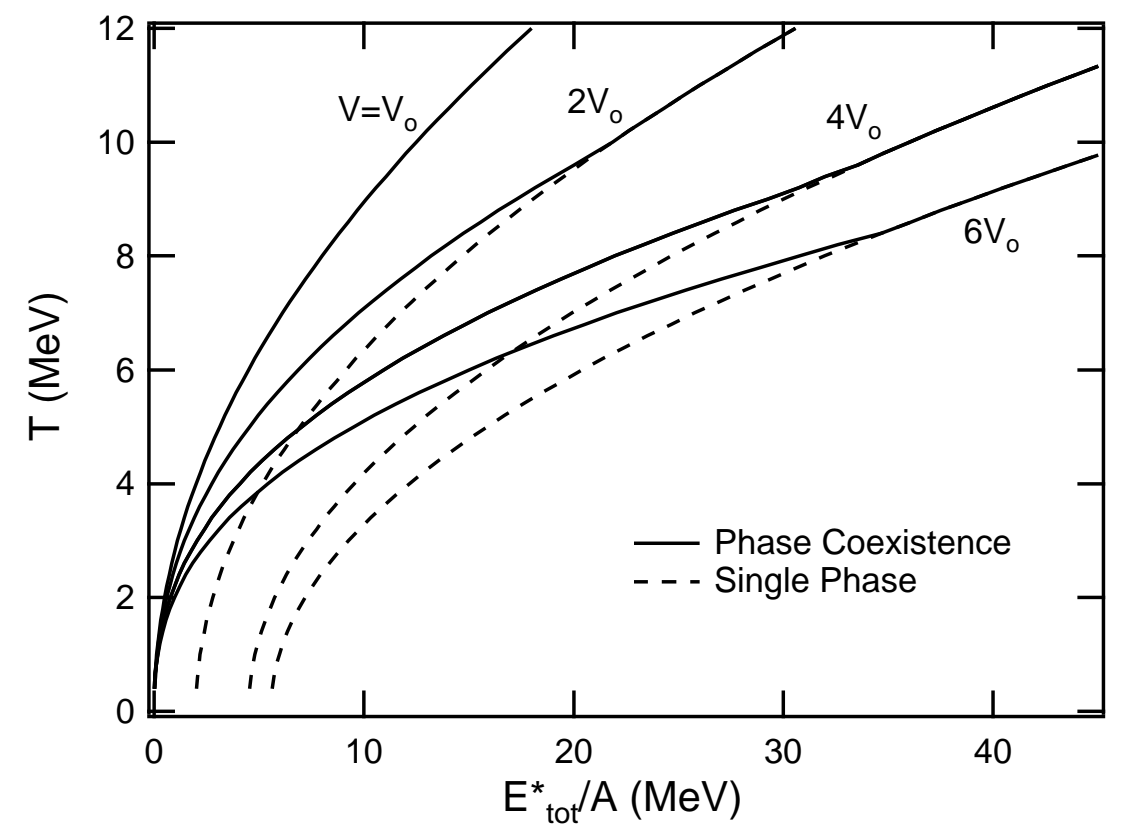

FIG. 3: Isochoric caloric curves for the boxed pseudo-Fermi matter, calculated for different volumes of the confining box, as indicated by labels.

this case, the temperature $T$ is plotted versus the matter density, for points along the boundary of the liquid-gas coexistence line (dashed line in Fig. 1). In the domain below this curve, the system is in a two-phase state. In this coexistence domain, the densities of gaseous and liquid phases at a given temperature are depicted by the intersection points of the coexistence curve with the horizontal line $T=$ const. On the left shoulder of the curve and in the domain further left to it, the system is in a pure gaseous state, while on the right shoulder and in the domain further right to it, the system is in a pure liquid state. The difference between liquid and gaseous phases vanishes for temperatures above $T=T_{c}=10.0 \mathrm{MeV}$.

The search for the minimum free energy for a given temperature $T$ and given volume $V$ yields, along with the volumes $V_{\text {gas/liquid }}$ and densities $\rho_{\text {gas/liquid }}$ of gaseous and liquid phases, the total excitation energy $E_{\text {total }}^{*}$ of the boxed matter. This allows one to construct the isochoric caloric curves for the modelled system at different volumes of the confining box. A set of such caloric curves is illustrated in Fig. 3. As expected, these curves do not feature plateaus reported in various experiments, [7] but rather inconspic- 
uous kinks at the locations on the boundary of the coexistence domain.

\section{CRITICAL BEHAVIOR}

One of the salient features of critical behavior in Van der Waals systems is the presence of a singularity at the critical point. Here, when the system temperature $\mathrm{T}$ approaches the critical temperature $T_{c}$ from below, the difference between the densities of coexisting liquid and gaseous phases vanishes according to a power law

$$
\rho_{\text {liquid }}-\rho_{\text {gas }}=C\left(T_{c}-T\right)^{\beta}
$$

In Eq. 17, $\mathrm{C}$ is a constant and $\beta$ is the critical exponent. The magnitude of the critical exponent can be extracted conveniently by fitting a straight line to a double-logarithmic

plot of $\left(\rho_{\text {liquid }}-\rho_{\text {gas }}\right)$ versus $\left(T_{c}-T\right)$. Such a plot obtained in present calculations is shown in Fig. 4. The plot features, indeed, an approximately $2-\mathrm{MeV}$ wide linear domain extending from the critical temperature $T_{c}$ down to lower temperatures. A linear fit to this domain allows one to extract the "coordinates" of the critical point of $T_{c}=10.0$ $\mathrm{MeV}$, and $\rho_{c} / \rho_{o}=0.42$. Furthermore, it yields a value of $\beta=0.51$ for the critical exponent.

Given the very schematic nature of the present formalism, the predicted characteristics of the critical point are well within a reasonable domain that can be inferred from more sophisticated, but less transparent calculations.

\section{DISCUSSION}

A simple formalism has been presented that allows one to model the behavior of interacting pseudo-Fermi matter under the conditions of controlled volume and temperature. The formalism is shown to capture the essential physics of a first-order liquid-gas phase transition. The calculation demonstrates the characteristics of nuclear liquid-gas coexistence in a certain domain of system parameters and the presence of a critical point. While such characteristics are well expected, based on the the similarity of the utilized form of interaction to that of the Van der Waals interaction, the present formalism offers many didactical benefits. For example, due to its simplicity it offers a clear insight into physical phenomena it purports to describe and a relatively strict test-bench for 


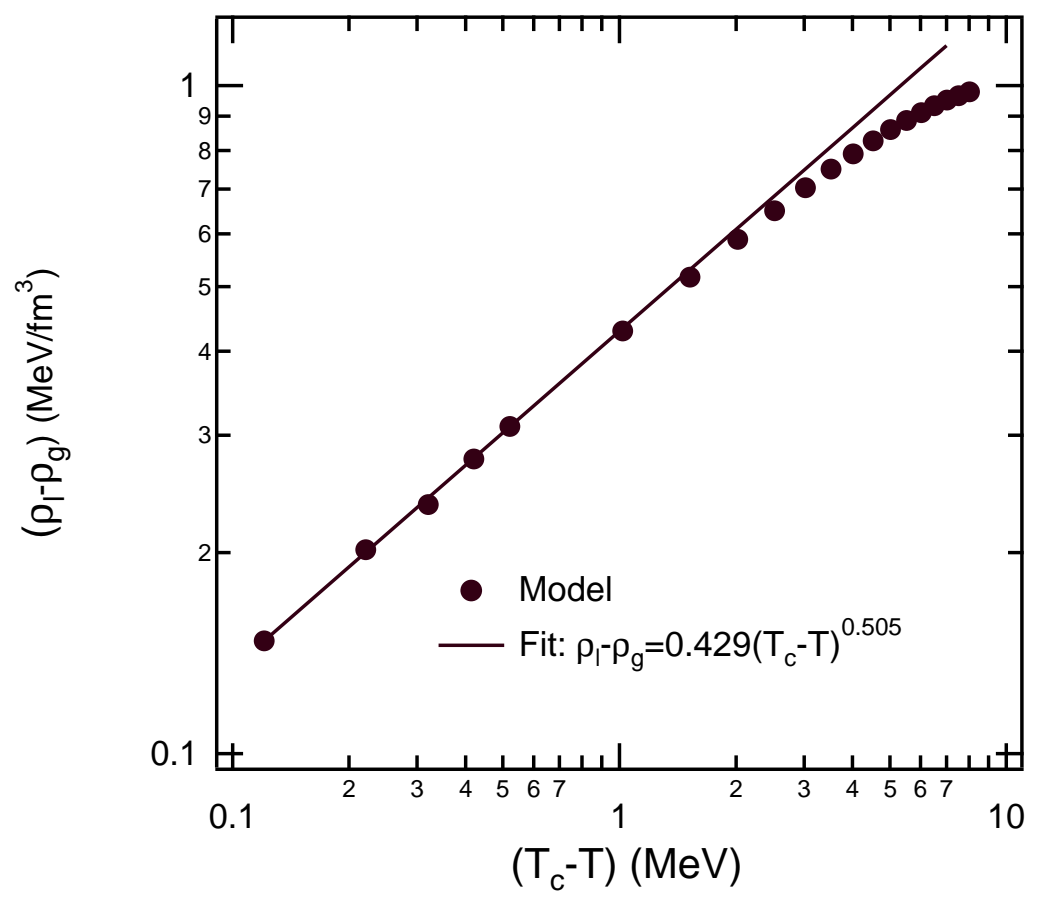

FIG. 4: Critical behavior of densities of coexisting liquid and gaseous phases at temperatures close to critical temperature $T_{c}$.

possible qualitative or "hand-waving" arguments. The results obtained, while almost trivial, may alert one to possible challenges found by more complete models. As an example, it is evident from Fig. 1 that models of nuclear multifragmentation such as SMM [2] and MMMC [1] tend to overpredict fragment multiplicities significantly. This is so, because these models assume that the liquid phase is always at ground-state density $\rho_{o}$. This forces the pressure of the surrounding gas within the freezeout volume to be higher than would be necessary to counter the lower pressure of the expanded liquid at equilibrium density. A higher gas pressure results in an overestimate of particle and fragment multiplicities.

The formalism presented here leaves ample room for further refinements such as a more strict modelling of the diluted Fermi matter, or the inclusion of isotopic effects. At any rate, it offers a convenient didactic tool to achieve a better understanding of nuclear thermodynamics. 


\section{Acknowledgments}

This work was supported by the U.S. Department of Energy grant No. DE-FG0288ER40414.

[1] D. H. E. Gross, Phys. Rep. 279, 119 (1997).

[2] J. P. Bondorf et al., Phys. Rep. 257, 133 (1995).

[3] J. Tõke and W. U. Schröder, Phys. Rev. C 65, 044319 (2002).

[4] J. Tõke and W. U. Schröder, Phys. Rev. Lett. 82, 5008 (1999).

[5] J. Tõke, J. Lu, and W. U. Schröder, This Annual Report, and submitted to Phys. Rev. C.

[6] W. A. Friedman, Phys. Rev. Lett. 60, 2125 (1988).

[7] J. B. Natowitz et al., Phys. Rev. C 65, 034618 (2002). 